\title{
Lexis
}

Journal in English Lexicology

13 | 2019

Lexicon, Sensations, Perceptions and Emotions

\section{L'expression de la perception visuelle : regard franco-japonais}

Aude Grezka et Aï Kijima

\section{(2) OpenEdition}

Journals

Édition électronique

URL : http://journals.openedition.org/lexis/3105

DOI : $10.4000 /$ lexis.3105

ISSN : 1951-6215

Éditeur

Université Jean Moulin - Lyon 3

Référence électronique

Aude Grezka et Aï Kijima, «L'expression de la perception visuelle : regard franco-japonais », Lexis [En ligne], 13 | 2019, mis en ligne le 14 mars 2019, consulté le 19 avril 2019. URL : http:// journals.openedition.org/lexis/3105; DOI : 10.4000/lexis.3105

Ce document a été généré automatiquement le 19 avril 2019

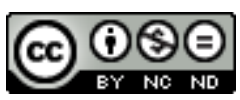

Lexis is licensed under a Creative Commons Attribution-NonCommercial-NoDerivatives 4.0 International License. 


\title{
L'expression de la perception visuelle : regard franco-japonais
}

\author{
Aude Grezka et Aï Kijima
}

\section{Introduction 1}

1 Il existe, dans toutes les langues, de nombreuses combinaisons lexicales pour exprimer une idée. En français, pour dire qu'une personne voit très bien, nous pouvons, entre autres, formuler les phrases suivantes :
(1) a. Léa a une vue perçante
b. Léa a une très bonne vue
c. Léa a des yeux de lynx
d. Léa voit comme un lynx
e. Léa a des yeux de chat

2 Les phrases (1c), (1d) et (1e) sont des expressions, des unités phraséologiques qui représentent le souhait du locuteur d'utiliser dans un acte de communication une formule, une suite lexicale connue et admise par la communauté linguistique, en se basant sur une valeur culturelle, sociale, générationnelle, diatopique, etc. au lieu d'utiliser une périphrase verbale traditionnelle comme en (1a) et (1b).

3 Ce phénomène se retrouve en japonais, bien que le système linguistique soit très différent de celui du français. Pour exprimer la même chose en japonais :
(2) 花子は目がいい2
Hanako ha me ga ii (Hanako voit bien / a de bons yeux)
Hanako / Th / yeux / S / bon

4 on pourra utiliser les expressions suivantes :

(3) a. 花子は目が利く

Hanako ha me ga kiku (Hanako a de bons yeux)

Hanako / Th / yeux / s / fonctionner 


\author{
b. 花子は目が肥えている \\ Hanako ha me ga koeteiru \\ Hanako / Th / yeux / S / être grossi \\ c. 花子は目が高い \\ Hanako ha me ga takai \\ Hanako / Th / yeux / s / être haut
}

Le fait d'utiliser ce type d'expressions, d'unités figées, implique deux choses : d'une part, le locuteur souhaite donner de la couleur à la langue et ainsi renforcer l'expressivité du sens (images mentales); d'autre part, les interlocuteurs doivent posséder un fonds culturel et linguistique commun pour pouvoir avoir une communication et une compréhension complète. Ainsi, la phrase Avec Orange, je vois rouge sera inintelligible pour l'interlocuteur s'il n'a pas à sa connaissance l'information que Orange désigne un opérateur téléphonique français, et que voir rouge est une expression pour dire « être en colère ».

6 Notre travail sur les prédicats de perception français et japonais (A. Grezka [2006, 2009] ; A. Kijima [2017]) nous a conduites à un constat. Les études linguistiques (monolingues, comparées, etc.) sur les verbes de perceptions sont nombreuses ${ }^{3}$ et mettent toutes en avant des difficultés liées à la syntaxe particulière et au sémantisme très riche, voire à la polysémie, de ces verbes. Mais si elles se sont principalement intéressées aux deux premiers phénomènes, force est de constater que certains traits ont peu retenu l'attention des linguistes, notamment le problème du figement, alors que les séquences figées sont pourtant nombreuses.

7 Cet article poursuit un triple objectif : faire un point sur la notion de figement en français et en japonais ; identifier les caractéristiques générales des verbes de perception visuelle dans ces deux langues; et enfin, étudier le traitement phraséologique des unités figées dans les dictionnaires français/japonais, à travers l'exemple de la perception visuelle.

\title{
1. Phraséologie et figement en France et au Japon : état de l'art
}

8 Dans cette section, nous faisons un rapide état de l'art de la phraséologie et du figement en France et au Japon.

\subsection{Le figement en France}

9 L'état actuel de la recherche en France dans le domaine de la phraséologie se caractérise par un débat théorique intense et un grand foisonnement terminologique, reflet d'une certaine dynamique interne. L'objet de la phraséologie soulève de nombreux questionnements.

Les Sciences du langage ont encore du mal à cerner et à définir ce phénomène complexe (J-C. Anscombre \& S. Mejri [2011]), particulièrement en Europe continentale (S. Mejri [2007] ; S. Granger \& M. Paquot [2008] ; D. Legallois \& A. Tutin [2013]) :

In scientific disciplines, it is customary to pay particular attention to the denomination of methodological concepts and to take the denomination as a pretext for theoretical discussions. The result of such a debate is usually conceptual precision, adequacy between the designated and the designator and the epistemological pertinence of the term being used. Frozenness is not an exception 
to the rule. As a phenomenon that is not yet clearly delineated, all the literature devoted to it has known a multitude of terms designating either the phenomenon or the product. As far as the phenomenon is concerned, the most common terms are phraseology, coalescence, idiomaticity, paremiology and frozenness. Such denominations stem from theoretical debates and approaches which, in the treatment of the phenomenon, favor certain aspects over others. (S. Mejri [2007 : 682])

11 Le figement a longuement été ignoré en France. Le statut de la question du figement et son évolution dans la théorie et dans la description des langues n'est pas à prendre à la légère :

En première étape, la syntaxe est avec Saussure le domaine de la parole et du discours où se place la liberté de l'individu dans les contraintes générales du système. De ce point de vue, il y a marginalisation des locutions et expressions. Elles présentent, certes, des traces diachroniques mais en synchronie le procédé mis en œuvre s'est bloqué en s'arrêtant à des variantes sémantiquement imprévisibles. Renvoyées à l'expressivité, à la figure, elles relèvent des formes stylistiquement enrichies. En deuxième étape, autour de la grammaire transformationnelle et générative, la syntaxe n'est plus le seul domaine de l'individu donc de la liberté. Les expressions idiomatiques, et d'une façon générale le figement, s'étudient avec les phrases libres. En syntaxe française deux articles de fond ont symbolisé ce tournant pris par les recherches linguistiques, à partir des travaux de Harris et Chomsky : Une classification des phrases "figées" du français (M. Gross 1982); Du bon usage des expressions idiomatiques dans l'argumentation en syntaxe générative (N. Ruwet 1983). En troisième étape, les expressions viennent sur le devant de la scène avec d'autant plus de poids que les outils informatiques permettent d'optimiser leurs procédures de reconnaissance dans les énoncés ainsi que leurs consultations dans des classements volumineux de type « lexiques-grammaires ». Les travaux nombreux et diversifiés conduisent à s'interroger avec une précision croissante sur la façon dont les facteurs de blocage pèsent sur les facteurs de liberté (et inversement) dans les productions langagières. Fondamentalement, la langue serait à considérer comme le lieu du figé, le libre apparaissant comme relativement marginal avec un continuum, et non plus du discret, du plus figé au libre, en passant par les collocations et le semi-figé. (A. Balibar-Mrabti \& C. Vaguer [2005 : 7-8])

12 Tout cela a entrainé un grand flottement terminologique qui traduit une importante dynamique terminologique : phrasèmes, unités phraséologiques, expressions figées, locutions, collocations, formules routinières, parémies, etc. M. Martins-Baltar avait déjà publié en 1997 une liste "nullement exhaustive» des dénominations que les linguistes français utilisaient dans le cadre de leur recherche en phraséologie: combinaison idiomatique, composé, construction, construction figée, énoncé lié, expression, expression idiomatique, expression proverbiale, expression semi-figée, façon de parler, fonction lexicale, forme de langage particulière, formulation, formule, fragment lié, etc.

Le figement est principalement défini par (G. Gross [1996]; R. Martin [1997] ; S. Mejri [1998] ; I. Gonzalez-Rey [2002] ; M-H. Svensson [2004]) :

- la non-compositionnalité sémantique ;

- la non-actualisation des référents ;

- la fixité syntaxique ;

- la restriction combinatoire.

Nous définissons ici le phénomène du figement au sens large (il serait en effet bien illusoire de pouvoir fournir une définition univoque). Nous entendons par expression figée une unité phraséologique constituée de plusieurs mots, contigus ou non, qui présentent un certain degré de figement sémantique, lexical et morphosyntaxique. 
Si l'on reprend la classification d'I. Gonzalez-Rey [2007], on peut distinguer trois grandes catégories dans les expressions figées :

- les expressions idiomatiques : dans lesquelles on trouve (i) les énoncés idiomatiques, c'est-àdire les expressions de la conversation quotidienne (formules routinières, expressions familières, onomatopées) ; (ii) les syntagmes idiomatiques, c'est-à-dire les expressions imagées groupées d'après des champs conceptuels ;

- les collocations ;

- les parémies (les proverbes et les expressions apparentées comme les sentences, les préceptes, les slogans, les devises, etc.).

\subsection{Le figement au Japon}

Du côté japonais, nous avons encore très peu de travaux sur le figement; ils sont marginalisés dans les études linguistiques au point d'être réduits à de simples remarques parcellaires. La phraséologie ou le figement ne constituent pas encore des sujets majeurs de la linguistique japonaise.

Comme pour la France, la terminologie sur le figement est très instable; plusieurs appellations sont utilisées sans être véritablement définies: 慣用表現 (expression idiomatique / idiome), 定型表現 (expression régulière), 凝結表現 (expression figée), 凍 結表現 (frozen expression), 成句 (locution), 相当句 (locution), etc. Les quelques définitions proposées diffèrent en plus en fonction des courants ou des écoles linguistiques: linguistique théorique, linguistique cognitive et linguistique japonaise.

Ainsi, chez les théoriciens, la fluence des expressions est importante, tandis que chez les linguistes qui s'intéressent à la langue japonaise, il y a une tentative de classement des différents types de séquences figées. Ce classement est cependant limité à trois hyperclasses: les proverbes, les collocations et les idiomes. Depuis les années 90 , on reconnaît, dans les idiomes, des sous-catégories comme la métaphore, la métonymie ou la synecdoque. Dans le domaine de la linguistique cognitive, les expressions idiomatiques sont plus traitées et analysées. Les cognitivistes différencient l'idiome du proverbe selon la forme et le sens et distinguent ensuite les expressions idiomatiques et les collocations du point de vue de l'opacité sémantique. Depuis P. Ishida [1999], les recherches sur le degré d'idiomaticité se multiplient. Pour vérifier le degré idiomatique des unités verbales, P. Ishida propose 6 étapes de transformations grammaticales propres à la langue japonaise: nominalisation (reconstruction des locutions), expressions passives (reconstruction des phrases), expressions impératives ou volontaires (substitution des éléments), addition ou insertion d'un modificateur aux éléments, négation ou affirmation (substitution des éléments) et addition ou insertion d'un modificateur aux phrases.

19 En résumé, les études sur les séquences figées au Japon ne dépassent pas celles du classement.

\section{Positionnement et avancée du projet}

Les travaux récents portant sur la phraséologie dans les langues, et par conséquent sur le figement, opèrent un recadrage qui met donc en évidence la centralité de cet aspect dans la description des langues. Loin d'être restreinte à des phénomènes linguistiques marginaux comme on l'a souvent pensé dans la tradition grammaticale et linguistique, la 
phraséologie intervient au sein même du fonctionnement des systèmes linguistiques. Elle agit comme un processus fondamental qui va assurer l'équilibre de l'économie générale des langues à côté de la liberté combinatoire. La phraséologie devient un élément central dans l'étude des langues faisant du lexique un point où se croisent prosodie, morphologie, syntaxe, sémantique et pragmatique.

21 Dans le cadre de notre projet, nous entendons par figement un certain degré de «fossilisation» d'une formation syntagmatique (P. Fiala et al. [1978] ; M. Gross [1982, 1988]; M-H. Viguier \& A. Grezka [2017]). La centralité du figement dans le fonctionnement des langues s'impose de plus en plus comme une évidence. Le figement est universel : toute langue vivante va exprimer sa vitalité, entre autres, par la création d'occurrences phraséologiques récurrentes. Les unités figées ont une place importante dans la maîtrise d'une langue. Une langue ne s'apprend pas uniquement par l'étude de ses règles grammaticales et de son lexique. La connaissance d'une langue se mesure également par la maitrise avec laquelle les usagers utilisent tous ces éléments figés représentatifs de chaque langue. Comme le signalent A. Rey \& S. Chantreau [2003 : VIII], «dans la pratique, ces notions [à propos des expressions et des locutions] sont essentielles; aucune langue ne peut s'apprendre, ni être décrite, sans elles ». Le seul fait de connaitre la signification de "voir » et de "jour » ne suffit pas pour comprendre l'expression voir le jour dans sa globalité. La citation de A. Rey \& S. Chantreau [2003 : VIII] étaye ce propos : l'expression est «un élément de la langue française qui fait partie du système même, du code ; qu'il faut donc maîtriser en tant que tel, et qu'il ne suffit pas de considérer comme un simple produit de règles syntactiques ou sémantiques». Les expressions forment la richesse imagée d'une langue, elles existent dans toutes les langues. Elles font partie de l'histoire et de la culture d'une langue et sont donc incontournables. Mais elles posent de nombreux problèmes : pour les locuteurs natifs qui, même s'ils les acquièrent naturellement au fur et à mesure de leur apprentissage, ne vont cependant pas être capables de les connaître dans leur totalité étant donné l'étendue du phénomène ; pour les apprenants d'une langue étrangère, qui vont se trouver face à de nombreuses combinaisons parfois opaques, voire indéchiffrables (P. Mogorrón Huerta [2010]).

22 Notre objectif est la mise en place d'une méthodologie de l'analyse et du traitement des phénomènes de figement en français et en japonais, et de proposer à terme une base de données de séquences figées franco-japonaises. La difficulté de ce travail réside principalement dans le fait que les travaux en phraséologie et sur le figement ne se situent pas au même niveau d'avancement dans les deux langues.

Ce travail est actuellement au stade exploratoire. Nous partons pour le moment de notre corpus sur la perception, pour tester notre modèle. Nous souhaitons dans ce travail de recherche explorer les multiples enjeux des recherches en phraséologie bilingue, tant du point de vue de la langue générale que de celui des langues de spécialité. Pour observer et comparer ces caractéristiques phraséologiques propres aux deux langues, nous souhaitons mettre en place un corpus parallèle recueillant des documents bilingues. Le dépouillement de ce corpus permettra de collecter et de modéliser des séquences figées selon une méthode développée pour les besoins de la recherche. L'analyse des données phraséologiques recueillies se fera à travers une approche syntactico-sémantique.

Dans la partie suivante, nous présentons les caractéristiques des verbes de perception visuelle en français et en japonais (fonctionnement, compléments, etc.). 


\section{3. Étude comparative : les verbes de perception visuelle}

La vue, d'un point de vue extralinguistique, est notre première source d'information, considérée comme objective, sur la réalité perçue. De ce fait, linguistiquement, le champ lexical relatif à la vision et à la vue est significativement plus abondant que celui des autres sens : on relève un grand nombre de noms, d'adjectifs, de verbes, d'expressions figurées et de locutions figées (A. Grezka [2006]). Cela se vérifie dans les deux langues.

\subsection{Caractéristiques générales des verbes de perception visuelle français}

Les verbes de perception visuelle expriment le fait de percevoir une réalité extralinguistique par le sens de la vue, c'est-à-dire à l'aide des organes sensoriels que sont les yeux. Il existe un ensemble assez important de verbes qui répondent à cette définition. Parmi ces verbes, on distingue ceux qui ont (i) une signification générale, comme voir, regarder et ceux qui véhiculent (ii) des nuances sémantiques plus subtiles, comme discerner, entrevoir, observer, etc.

Voir et regarder sont considérés dans la littérature comme les verbes fondamentaux et prototypiques de la perception visuelle. Cette représentativité est d'ailleurs illustrée par la liste de fréquence lexicographique du Ministère de l'Éducation nationale, de l'enseignement supérieur et de la recherche ${ }^{4}$. Voir se situe au $7^{\mathrm{e}}$ rang des verbes français, après les verbes être, avoir, faire, dire, pouvoir et aller. Quant à regarder, on le retrouve à la $17^{\mathrm{e}}$ place sur 421 verbes. Cette liste de fréquence lexicographique permet aux enseignants, à partir d'un corpus de vocabulaire de base du français, de bâtir des progressions qui répondent aux objectifs d'apprentissage fixés pour leurs élèves.

Si les verbes voir et regarder dénotent un même type de perception sensorielle, ils n'ont pourtant pas les mêmes propriétés linguistiques. Ce qui différencie ces deux verbes prototypiques, c'est l'intentionnalité ou non du sujet, c'est-à-dire son attitude passive ou active par rapport au procès.

Le verbe voir est susceptible de recevoir différents types d'interprétation déterminés en partie par le type de ses arguments et de son contexte. Ce verbe a de nombreuses propriétés qui le distinguent de tous les autres verbes de perception. Syntaxiquement, voir apparaît dans de nombreuses constructions qui n'existent pas pour les autres verbes de perception. On le rencontre même en position de semi-auxiliaire dans des constructions comme Il s'est vu fermer la porte au nez. Morphologiquement, le nombre d'affixes et de composés pouvant s'ajouter à voir est plus important que celui des autres verbes de perception (prévoir, entrevoir, pourvoir, etc.). Enfin, le verbe a un champ sémantique assez vaste, plus large en tout cas que celui des autres verbes de perception puisque nombre de ses emplois se rapportent à des facultés cognitives.

Les emplois perceptifs de voir se scindent en trois groupes: perception physiologique, physio-cognitive ou cognitive. Les emplois de voir qui ressortissent à la perception physiologique ont un lien direct avec le sens visuel, ce qui n'est pas le cas des emplois cognitifs pour lesquels, la perception passe par l'esprit. Quant aux emplois de voir qui sont rattachés à la perception physio-cognitive, la perception est à mi-chemin entre le 
physiologique et le cognitif (inférence fondée sur des données visuelles), puisque le sujet interprète une situation en tirant des conclusions de tout ce qui se présente à ses yeux. Ces oppositions ont donné lieu à une répartition des emplois de voir en trois hyperclasses :

(4) J'ai vu l'accident

= Prédicats de <perception physiologique>

(5) Je vois que tu as cassé le vase

= Prédicats de <perception physio-cognitive>

(6) Je vois ton problème

$=$ Prédicats de <perception cognitive $>$

31 Cette complexité du traitement des emplois de voir s'explique par la nature même de la polysémie: elle est de type interne. Les emplois perceptifs de voir présentent des extensions de sens nombreuses. Cette polysémie concerne des extensions à l'intérieur même de la perception. Le système de la perception visuelle se laisse structurer selon les quatre principaux axes suivants :

- passive (voir un accident) /v/ active (voir un match)

- directe (voir quelqu'un se lever) /v/ indirecte (voir à sa tête que quelqu'un est rentré tard)

- réelle (voir un ami) /v/ imaginée (voir un ami en rêve)

- physiologique (voir un ballon) /v/ cognitive (voir un problème)

Comme le signale H. Bat-Zeev Shyldkrot [1989 : 288] :

Étant donné que nous empruntons souvent des concepts du monde physique, qui nous est plus accessible, pour exprimer des notions intellectuelles et émotives relatives à un monde plus abstrait, il n'est pas étonnant que voir couvre beaucoup de sens liés aux facultés intellectuelles ou au savoir, relevant du domaine objectif et non pas du domaine subjectif. Le domaine intellectuel, objectif est compris comme distant alors que le subjectif est plutôt perçu comme proche.

Le verbe regarder n'embrasse pas quant à lui la même étendue sémantique que le verbe voir. La liste des différentes significations rattachées aux deux verbes de perception montre que les verbes exprimant généralement une perception passive (voir) sont susceptibles d'une plus grande variété sémantique que ceux exprimant une perception active (regarder). Parmi les 11 emplois de regarder, seulement 7 appartiennent au domaine de la perception. Alors qu'il est possible avec le verbe voir de passer d'une lecture "passive " (i.e. perception non intentionnelle) à une lecture "active» (i.e. perception intentionnelle), l'inverse n'est pas vrai pour le verbe regarder. Les emplois perceptifs de regarder ne peuvent exprimer qu'une perception intentionnelle. Ces impossibilités expliquent en partie les restrictions observées sur le champ sémantique de regarder. Du point de vue syntaxique, le verbe regarder est également beaucoup moins productif: il apparait dans un nombre bien plus limité de constructions que le verbe voir.

\subsection{Caractéristiques générales des verbes de perception visuelle japonais}

Comme pour le français, de nombreux verbes japonais expriment la perception visuelle. Le verbe principal pour la perception visuelle est miru (cf. A. Kijima [2014, 2016]). Ce verbe a la caractéristique de pouvoir s'écrire de différentes manières; ses idéogrammes sont variés : 見,観, 視,診,看 et 覧. L'idéogramme 見 est statistiquement le plus fréquemment 
utilisé6. Par ailleurs, nous le retrouvons comme radical dans la construction de nombreuses expressions verbales telles que :

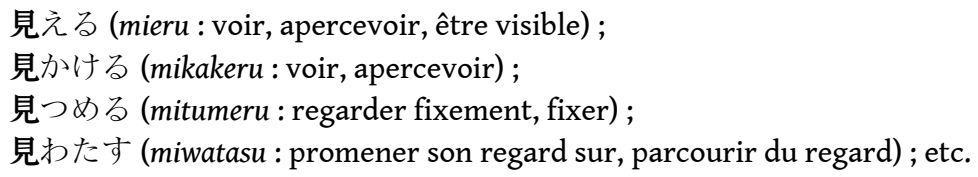

Miru peut également être accompagné d'adverbes qui le nuancent: ちらっと見る ( chiratto-miru : entrevoir), じっと見る (jitto-miru : regarder fixement, fixer),かいま見る( kaima-miru : entrevoir, apercevoir), しげしげと見る (shigeshigeto-miru: dévisager).

Ces diverses caractéristiques de miru (見る) confirment bien l'importance de ce verbe dans la langue japonaise. C'est pourquoi nous le mettons au centre de notre étude. En japonais, miru est classé parmi les verbes dits d'activité externe. En effet, selon M. Kudo [1995], on peut diviser les verbes japonais en trois catégories: activité externe, état interne et état statique. Les verbes d'activité externe sont ensuite sous-divisés à l'aide des critères suivants : continuité, instant, subjectivité et objectivité ; propriétés sémantiques : activité du sujet et changement du sujet. Miru appartient aux verbes d'activité externe du fait de l'activité du sujet sans limite interne : continuité de l'activité (de manière active et passive).

Le verbe miru est presque toujours analysé de pair avec mieru car ce dernier verbe est un dérivé de miru auquel a été adjoint le verbe auxiliaire de volonté $y u^{7}$. Or, les constructions syntaxiques de ces verbes sont différentes ${ }^{8}$ :

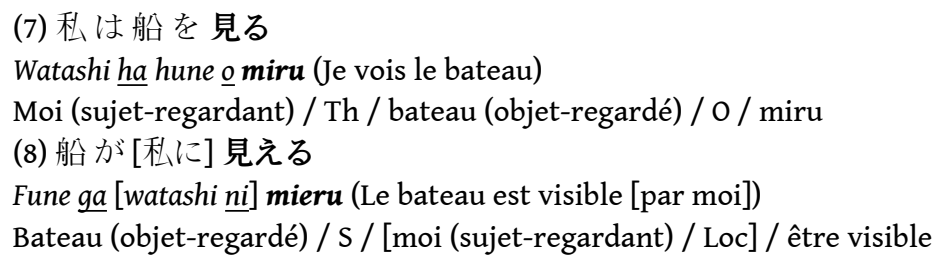

Mieru en (8) désigne le fait d'être visible à l'instant présent (c'est-à-dire sans effort particulier, sans chercher à voir). Le bateau est visible car la vue est dégagée ou parce qu'il arrive soudainement dans mon champ de vision. À l'inverse, dans l'exemple (7) avec miru, je vois le bateau car je cherche à le voir.

Le verbe japonais miru est connu pour sa polysémie - tout comme le verbe voir en français. Plusieurs chercheurs ont proposé des classements sémantiques de ce verbe. Ainsi, l'étude de S. Tanaka [1996, 1999], la plus récente et la plus détaillée, isole 4 des nombreuses définitions habituellement trouvées dans les dictionnaires. Elle distingue un sens « fondamental », c'est-à-dire la vision active, trois sens dérivés « interprétatifs », un sens dérivé « métaphorique » et un dernier sens « métonymique » :

A. SENS FONDAMENTAL : reconnaître l'objet par la vue

B. SENS DÉRIVÉS PAR L'INTERPRÉTATION :

a. Juger et interpréter l'objet en se basant sur la reconnaissance visuelle

b. Juger et interpréter l'objet

c. Juger et interpréter visuellement l'objet et l'analyser convenablement

C. SENS MÉTAPHORIQUE: juger et interpréter l'objet en se basant sur une

reconnaissance autre que visuelle

D. SENS MÉTONYMIQUE : faire l'objet de / se produire (pour une situation) 
Si nous classons tous les emplois de miru en appliquant les critères syntacticosémantiques de A. Grezka [2009], nous obtenons les 13 emplois suivants :

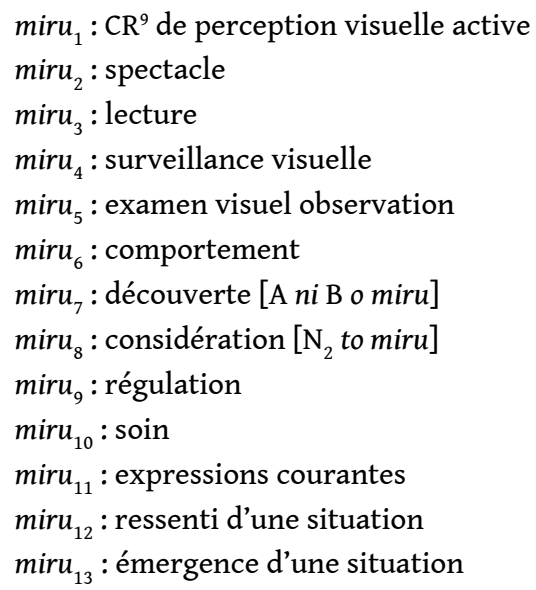

\section{3. Étude comparative des verbes de perception français/japonais}

Pour ce travail, nous nous sommes appuyées sur la grille d'analyse proposée par A. Grezka [2006, 2009], qui a servi à dégager les divers emplois des verbes de perception français, voir et regarder. Cette grille a été élaborée dans le but de décrire systématiquement les verbes de perception et de les classer sur la base de leurs propriétés communes. Elle est constituée de cinq catégories générales de propriétés linguistiques : propriétés configurationnelles, sémantiques, combinatoires, syntaxiques et morphologiques. Cette grille est strictement adaptée à l'étude des prédicats verbaux : la polysémie des verbes constitue l'un des problèmes les plus ardus de la description des langues parce qu'elle met en jeu un tissu de relations complexes. Les emplois d'un verbe donné sont régis par des facteurs profondément hétérogènes, d'ordre sémantique, syntaxique et lexical mais également aspectuo-temporel. Pour le verbe voir, A. Grezka distingue 17 emplois et 11 pour le verbe regarder. Ces emplois ont été répartis dans des classes sémantiques (la justification de ces classes procède de propriétés syntacticosémantiques; chacune de ces classes a sa propre combinaison de traits) : 5 classes pour voir et 2 classes pour regarder:

Tableau 1 : Classement des verbes visuels en français

\begin{tabular}{|l|l|}
\hline 1. vision passive & $\begin{array}{l}\text { capacité visuelle, acuité visuelle, } \mathrm{CR} \text { de perception visuelle passive, } \\
\left.\text { propriété visuelle d'un lieu / objet ( } \text { voir }_{1-4}\right) \\
\text { Ex. Léa a vu un homme dans le jardin; De la terrasse, on voit le Vésuve }\end{array}$ \\
\hline 2. vision active & $\begin{array}{l}\text { CR de perception visuelle active, indication, spectacle, lecture, recherche, } \\
\text { surveillance visuelle, examen visuel, visite, perception physio-cognitive } \\
\left(\text { voir }_{5-9}, \text { regarder }_{1-7}\right) \\
\text { Ex. Léa a vu hier soir le match de foot ; Léa regarde le paysage }\end{array}$ \\
\hline 3. représentation & $\begin{array}{l}\left.\text { illusion, imagination, divination/prévision ( } \text { voir }_{10-12}\right) \\
\text { Ex. Je vous ai vu cette nuit en rêve }\end{array}$ \\
\hline
\end{tabular}




\begin{tabular}{|c|c|}
\hline 4. intellection & $\begin{array}{l}\text { constatation, considération, jugement, conception, compréhension, } \\
\text { comportement ( } \operatorname{voir}_{13-17}, \text { regarder }_{8-9} \text { ) } \\
\text { Ex. Je verrai le problème avec lui }\end{array}$ \\
\hline $\begin{array}{l}\text { 5. opération } \\
\text { sociale }\end{array}$ & $\begin{array}{l}\text { voir }_{18} \text { voir }_{19} \text { voir }_{20} \text { voir }_{21} \\
\text { Ex. Léa a vu un médecin pour son mal de gorge }\end{array}$ \\
\hline 6. intérêt & $\begin{array}{l}\text { regarder }_{10} \\
\text { Ex. Cette affaire te regarde }\end{array}$ \\
\hline 7. orientation & $\begin{array}{l}\text { regarder }_{11} \\
\text { Ex. La maison regarde la mer }\end{array}$ \\
\hline
\end{tabular}

En appliquant cette classification aux emplois japonais des verbes de perception, nous obtenons les concordances suivantes :

Tableau 2 : Comparaison des emplois

\begin{tabular}{|c|c|c|}
\hline 1. vision passive & représenté par le verbe mieru & \\
\hline \multirow{5}{*}{ 2. vision active } & miru $_{1}$ (CR de perception visuelle active) & regarder $_{1}$ \\
\hline & $\operatorname{miru}_{2}:$ spectacle & voir $_{5}$, regarder $_{3}$ \\
\hline & $\operatorname{miru}_{3}:$ lecture & voir $_{6}$, regarder $_{4}$ \\
\hline & miru $_{4}$ : surveillance visuelle & regarder $_{6}$ \\
\hline & miru $_{5}:$ examen visuel & $\operatorname{voir}_{7}$, regarder $_{7}$ \\
\hline 3. représentation & \multicolumn{2}{|l|}{ représenté par le verbe mieru } \\
\hline \multirow{5}{*}{ 4. intellection } & miru $_{6}:$ comportement & regarder ${ }_{9}$ \\
\hline & miru $_{7}$ : découvert: $[$ A ni B o miru] & \\
\hline & miru $_{8}$ : considération $:\left[\mathrm{N}_{2}\right.$ to miru $]$ & voir $_{14}$, regarder $_{8}$ \\
\hline & miru $_{9}:$ régulation & accorder, régler \\
\hline & $\operatorname{miru}_{10}:$ soin & s'occuper de \\
\hline Expressions & $\begin{array}{l}\operatorname{miru}_{11}: \text { expressions courantes } \\
\text { miru }_{12}: \text { ressenti d'une situation } \\
\text { miru }_{13}: \text { émergence d'une situation }\end{array}$ & \\
\hline
\end{tabular}

Parmi les sept classes observées dans le tableau 1, seules les classes vision passive et représentation sont équivalentes au verbe mieru. Les autres classes emploient le verbe miru. 
Quant aux classes opération sociale, intérêt et orientation, elles n'utilisent pas un verbe de perception visuelle, contrairement au français.

Nous allons étudier dans notre dernière partie la question du figement dans notre corpus bilingue sur la perception. Les séquences figées sont nombreuses mais, comme nous allons le voir, leur extraction est particulièrement difficile car le traitement phraséologie des unités figées dans les dictionnaires français/japonais est problématique.

\section{Les expressions visuelles figées}

Notre expérience d'enseignant et de chercheur en phraséologie (monolingue et bilingue), nous a permis d'observer :

- l'importance du figement dans le lexique et dans les situations discursives quotidiennes ;

- le traitement insuffisant donné par les dictionnaires (monolingues et bilingues) aux unités phraséologiques, avec des difficultés évidentes à trouver des équivalences sémantiques acceptables dans une autre langue

- l'absence d'un système efficace de sélection des unités figées présentes dans les manuels d'enseignement du Français Langue Étrangère ou du Japonais Langue Étrangère.

\subsection{Le traitement phraséologique des unités figées dans les dictionnaires monolingues/bilingues}

Le premier réflexe lorsqu'un usager se trouve face à une expression figée qui n'est pas de sa compétence linguistique, c'est de la rechercher dans un dictionnaire afin d'être capable de l'interpréter correctement ou, s'il s'agit d'une langue étrangère, de pouvoir la traduire. Mais on relève de nombreux problèmes présents aussi bien dans les dictionnaires français que dans les dictionnaires japonais. Premièrement, les unités figées retenues sur contextes et recherchées sont fréquemment absentes de ces ouvrages. Les dictionnaires monolingues et bilingues n'incorporent dans leurs fonds lexicographiques qu'un nombre assez restreint d'unités figées.

Deuxièmement, on observe un déséquilibre important entre le processus de renouvellement de la langue et le matériel que les lexicographes incorporent dans les dictionnaires. Nous avons des unités figées de langue classique, fréquemment hors d'usage, qui ne sont plus représentatives de l'usage contemporain de la langue.

Par ailleurs, les unités figées retenues par un dictionnaire ne sont pas les mêmes d'un dictionnaire à un autre. On peut donc se poser la question de savoir quels sont les critères de sélection des unités figées. Pourquoi telle expression plutôt qu'une autre?

De même, les variations d'une unité figée ne sont pas toutes référencées dans le dictionnaire et peuvent même être différentes entre deux dictionnaires. Prenons l'exemple des dictionnaires français monolingues. Si l'on recherche les expressions relatives au fait de "voir très bien ", on trouvera respectivement dans les dictionnaires Larousse, Le Robert et le Trésor de la Langue Français informatisé $(T L F i)^{10}$ les unités suivantes :

- Larousse : avoir un œil de lynx

- Le Robert : avoir des yeux, un ceil d'aigle

- TLFi : (œil, regard, vue) de lynx

Nous obtenons donc des résultats très différents en fonction du dictionnaire. On remarquera d'ailleurs qu'il n'y a que dans le dictionnaire Le Robert que l'on trouve les 
expressions à l'entrée voir ; pour les autres dictionnaires, nous avons dû aller chercher les expressions sous l'entrée ceil. Nous avons également très peu d'informations sur leur utilisation, aussi bien sémantiques, syntaxiques que morphologiques. Par exemple, ces expressions peuvent-elles être utilisées avec un sujet humain et/ou animal ? Peut-on les mettre au pluriel ?, etc.

Enfin, un problème que nous rencontrons assez souvent dans les dictionnaires bilingues, notamment japonais/français, est le fait de classer des phrases comme expressions alors qu'elles ne sont pas figées. On trouve ainsi dans le dictionnaire 仏和辞典 (futsu-wa Jiten ${ }^{11}$ ), les phrases suivantes classées comme expressions :

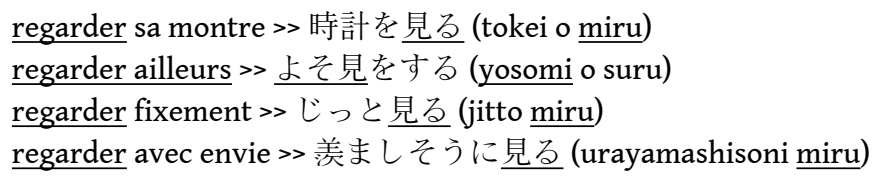

Il existe concrètement très peu de dictionnaires bilingues français-japonais (le japonais est plus souvent comparé avec l'anglais, le coréen, le chinois et de temps en temps avec l'allemand et le russe). Ceux-ci ont le plus souvent des dictionnaires « grand public », non linguistiques.

\subsection{Perception visuelle et séquences figées}

Nous avons donc essayé de répertorier l'ensemble des séquences figées, construites à partir d'un verbe, d'un nom ou d'un adjectif de perception visuelle. Dans les deux langues, le figement est assez conséquent. Pour le français, les séquences figées se répartissent de la manière suivante :

Figure 1 : Séquences figées françaises

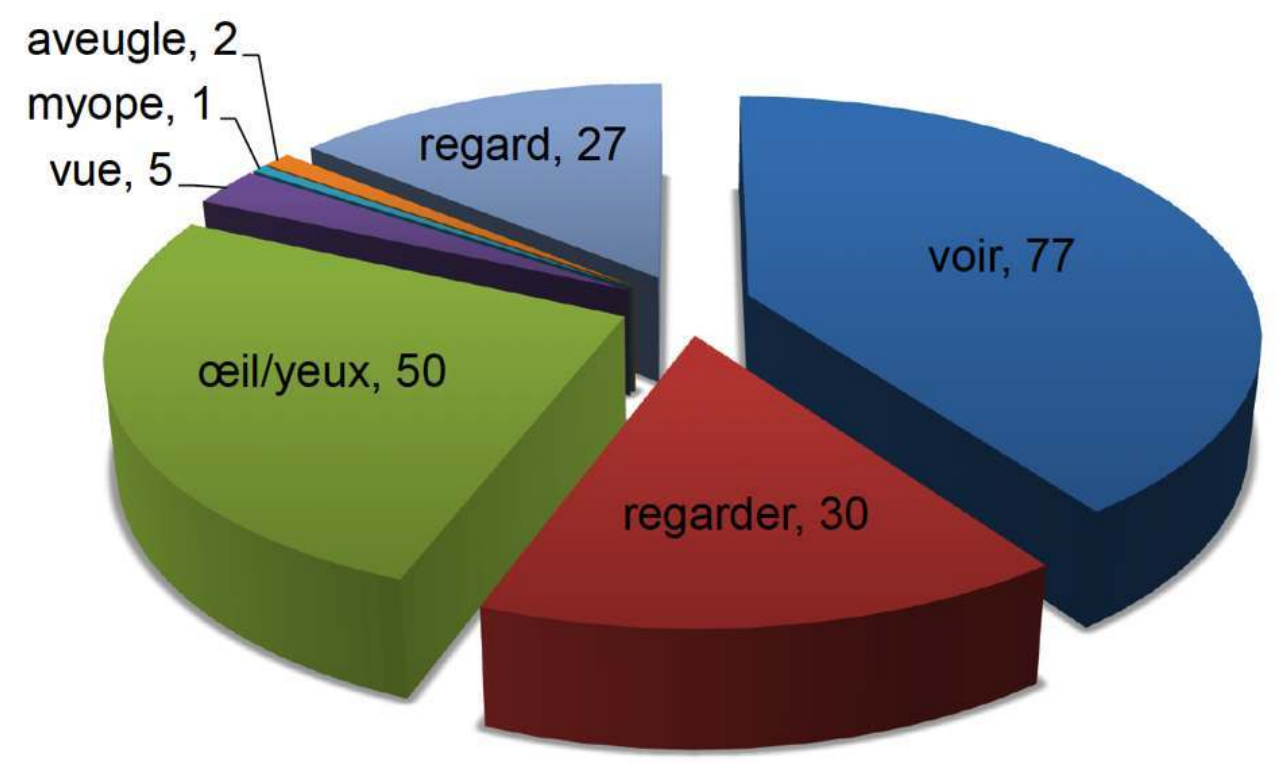

Les expressions construites à partir du verbe de perception voir sont les plus nombreuses. Certaines sont propres à la perception visuelle comme : 
Avoir des yeux de lynx, d'aigle

Se regarder en chien de faïence

Être myope comme une taupe

Adresser un regard double sens en fonction du contexte (regarder en face, regarder en dessous, etc.). Si l'expression regarder en dessous désigne dans son sens premier une personne regardant de manière oblique vers le bas, baissant donc les yeux par signe de respect ou de timidité, elle peut également être attribuée à quelqu'un de sournois cherchant à fuir le regard de son interlocuteur. Enfin, certaines expressions prennent un tout autre sens, sans lien avec la perception visuelle, bien qu'associées à un verbe de perception visuelle : voir rouge, noir ; avoir vu le loup ; regarder les mouches voler ; avoir un regard d'aigle ${ }^{12}$.

Pour les séquences figées japonaises, un grand nombre d'entre elles sont construites à partir du nom me (œeil, yeux) ou de ses synonymes (par exemple manako, katame, hitomi), le verbe miru vient en seconde position. Le verbe a une place moins importante qu'en français :

(9)目を光らす

Me wo hikarasu (garder un œil vigilant sur quelque chose / avoir l'œil sur)

œil / o / faire briller

Figure 2 : Séquences figées japonaises

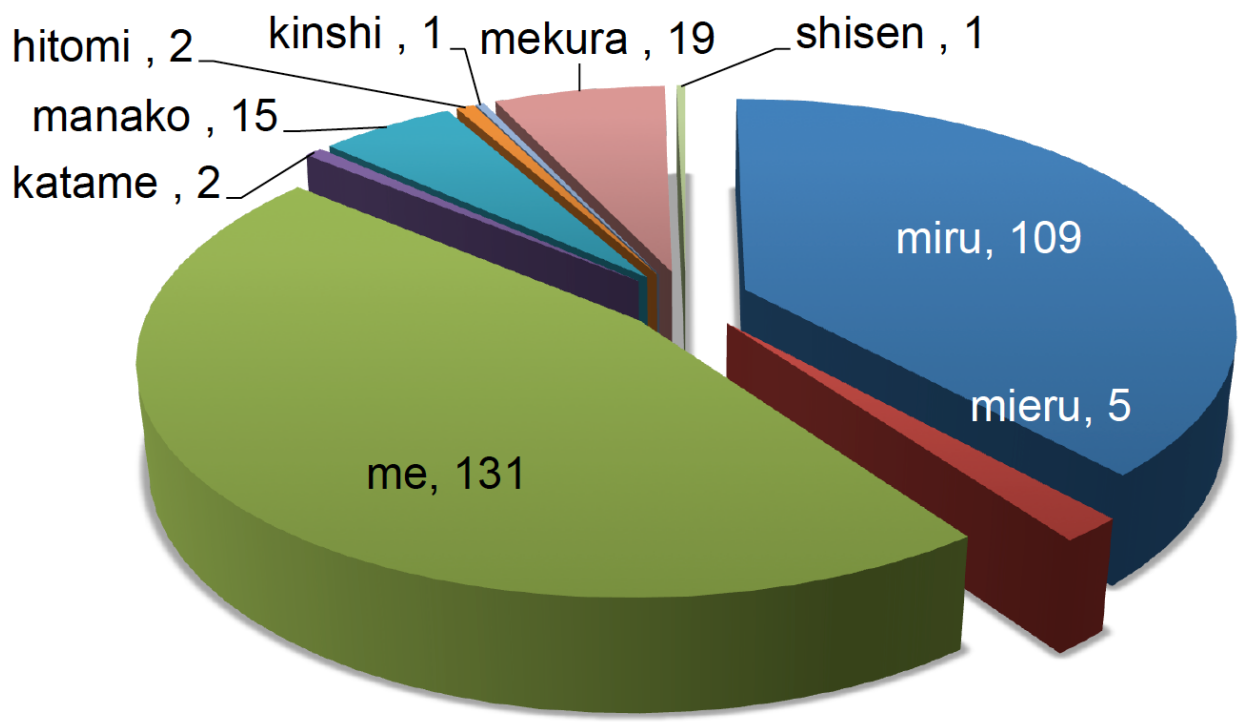

Nous pouvons résumer l'ensemble de nos résultats par le tableau suivant :

Tableau 3 : Les éléments visuels dans les séquences figées

\begin{tabular}{|l|l|l|l|l|}
\hline \multicolumn{1}{|l|}{ FRANÇAIS } & \multicolumn{2}{l|}{ JAPONAIS } \\
\hline \multirow{2}{*}{ VERBE } & voir & 77 & miru & 109 \\
\cline { 2 - 5 } & regarder & 30 & mieru & 5 \\
\hline
\end{tabular}




\begin{tabular}{|c|c|c|c|c|}
\hline \multirow{8}{*}{ NOM } & \multirow{4}{*}{ œil/yeux } & \multirow{4}{*}{50} & me & 131 \\
\hline & & & manako & 15 \\
\hline & & & katame (un œil) & 2 \\
\hline & & & hitomi (prunelle/œil) & 2 \\
\hline & vue & 5 & pas d'équivalence & \\
\hline & myope & 1 & kinshi & 1 \\
\hline & aveugle & 2 & mekura & 19 \\
\hline & regard & 27 & shisen & 1 \\
\hline
\end{tabular}

\section{Conclusion}

Qu'il s'agisse de lexicographie, de didactique des langues ou de traitement automatique des langues, on réalise aujourd'hui que la prise en compte des phénomènes phraséologiques constitue un problème qu'il est temps d'examiner. C'est dans cette perspective applicative que cet article a eu pour objectif de rendre compte des travaux récents en français et en japonais sur le figement, par le biais des verbes de perception.

Les résultats que nous obtiendrons dans le cadre de notre projet permettront une meilleure compréhension du fonctionnement du niveau phraséologique de la langue française et japonaise. Ces résultats trouveront des applications concrètes notamment dans le cadre de la création d'outils d'aide à la rédaction et de l'apprentissage des langues. En effet, aux débats théoriques font écho les interrogations des didacticiens, traducteurs et informaticiens qui, confrontés à la complexitê du fait phraséologique, y ont vu l'une des difficultés majeures dans l'apprentissage des langues, notamment étrangères, dans la traduction des textes et dans le traitement automatique. Ainsi, dans le domaine didactique, il faut rappeler la longue tradition lexicographique et les traitements réservés aux séquences figées, les manuels et les méthodes d'enseignement élaborés dans le cadre de plusieurs projets. Dans le domaine de la traduction, la présence importante des unités syntagmatiques et leur impact dans les traductions proposées. Enfin, en informatique, le point essentiel demeure la reconnaissance et la génération automatique des unités polylexicales.

\section{BIBLIOGRAPHIE}

ANSCOMBRE Jean-Claude \& MEJRI Salah (eds.), 2011, Le figement linguistique : la parole entravée, Éditions Honoré Champion, collection « Lexica, Mots et Dictionnaires ", n¹8. 
BAlibAR-MRABTI Antoinette \& VAGUeR Céline, 2005, « Présentation. Le semi-figement », Linx 53, 7-15, également disponible à : http://journals.openedition.org/linx/252

BAT-ZEEV SHYLDKROT Hava, 1981, « À propos de la forme passive se voir + Vinf. », Folia Linguistica XV : 3-4, 387-408.

BAT-ZEEV SHYLDKROT Hava, 1989, « Les verbes de perception : étude sémantique », in KREMER Dieter (ed.), Actes du XVIIe congrès International de linguistique et philologie romanes, Tome 4, Tübingen : Max Niemeyer Verlag, 282-294.

DANNELL Karl Johan, 1992, "Nothing but phrases. About the distribution of idioms and stock phrases", in EDLUND Lars-Erik \& PERSSON Gunnar (eds.), Language - the Time Machine. Papers in honour of Bengt Odenstedt on the occasion of his sixtieth birthday, July 21, 1992, Stockholm : Almqvist \& Wiksell, 17-29.

DUBoIS Jean \& DUBOIS-CHARLIER Françoise, 1997, Les verbes français, Paris : Larousse.

FIALA Pierre, HABERT Benoît, LAFon Pierre \& PINEIRA-TRESMONTANT Carmen, 1978, « Des mots aux syntagmes. Figement et variation dans la Résolution générale de la CGT de 1978 », Mots 14, 47-88. FRANCKEL Jean-Jacques, 1989, Étude de quelques marqueurs aspectuels du français, Genève : Droz. FRANCKEL Jean-Jacques \& LEBAUD Daniel, 1990, Les figures du sujet. À propos des verbes de perception sentiment, connaissance, Paris : Ophrys.

GONZALEZ-REY Isabel, 2002, La phraséologie du français, Toulouse : Presses Universitaires du Mirail. GONZALEZ-REY Isabel, 2007, La didactique du français idiomatique, Fernelmont (Belgique) : E.M.E. Éditions.

GRANGER Sylviane \& PAQUOT Magali, 2008, "Disentangling the phraseological web", in GRANGER Sylviane \& MEUNIER Fanny (eds.), Phraseology: An Interdisciplinary Perspective, Amsterdam: John Benjamins, 27-49.

GREZKA Aude, 2006, Les prédicats de perception. Traitement de la polysémie, Thèse de doctorat en Sciences du langage, Université Paris 13.

GREZKA Aude, 2009, La polysémie des verbes de perception visuelle, Paris : L'Harmattan, Collection 'Sémantiques'.

GREZKA Aude, 2016, «Classes et relations sémantiques : l'exemple du verbe regarder », Neophilologica 28, 72-97.

GROSS Gaston, 1996, Les expressions figées en français : noms composés et autres locutions, Paris : Ophrys.

GRoss Gaston, 2012, Manuel d'analyse linguistique, Villeneuve d'Ascq : Presses Universitaire du Septentrion.

GROSS Maurice, 1982, « Une classification des phrases figées du français », Revue québécoise de linguistique, Vol. 11, $\mathrm{n}^{\circ} 2,151-185$.

GROSS Maurice, 1988, Grammaire transformationnelle du français. 3. Syntaxe de l'adverbe, Paris : Cantilène.

ISHIDA Priscilla, 1999, «Syntactic Frozenness as a Criterion for Measuring the Degree of Idiomaticity of Verb Idioms », Tsukuba journal of applied linguistics 6, 69-83.

KIJIMA Aï, 2014, «Considérations sur le verbe visuel japonais miru », Bulletin de linguistique et de littérature françaises de l'Université de Tsukuba 29, 142-154. 
KIJIMA Aï, 2016, «Considérations sur l'expression figée n'avoir rien à voir », Linguistique de la parole, Hors-série du Bulletin d'études de linguistique française 50, 51-70.

KIJIMA Aï, 2017, Étude comparée des verbes de perception visuelle en français et en japonais, Thèse de doctorat en Linguistique, Université de Franche-Comté.

KUDO Mayumi, 1995, Aspect-Tense Systems and Text: Temporal Expressions in Modern Japanese, Tokyo: Hituji Syobo.

KUNIHIRO Tetsuya, 1985, «La théorie des expressions figées », Lingistics japanises 4, 4-14.

LEGALLoIs Dominique \& TUTIN Agnès, 2013, « Présentation : Vers une extension du domaine de la phraséologie ", Langages 189, 3-25.

LE GOFFIC Pierre, 1993, Grammaire de la phrase française, Paris : Hachette.

MARTIN Robert, 1997, « Sur les facteurs du figement lexical », in MARTINS-BALTAR Michel (ed.), La locution entre langue et usages, Paris : ENS Éditions / Ophrys, 291-305.

MARTINS-BALTAR Michel, 1997, La locution, entre langue et usages, Paris : ENS Éditions / Ophrys.

MEJRI Salah, 1998, «La conceptualisation dans les séquences figées », L'Information Grammaticale 2, Numéro spécial Tunisie, 41-48.

MEJRI Salah, 2007, "French phraseology", in BURGER Harald, DOBRovol'SKIJ Dmitrij, KÜHN Peter \& NORRICK Neal R. (eds.), Phraseologie / Phraseology. Ein internationales Handbuch der zeitgenössischen Forschung / An International Handbook of Contemporary Research, Berlin/New York: Walter de Gruyter, 682-690.

MIYAJI Yutaka, 1982, L'emploi et la signification des expressions figées, Japon : Meiji-shoin.

MIYAJIMA Tatsuo, 1972, A Descriptive study on the meaning and use of Japanese Verbs, Syuei-syuppan: The National Language Research Institute Research Report 43, 654-664.

MOGORRÓN HUERTA Pedro, 2010, «Peut-on traduire les expressions figées », Les Cahiers du CENTAL 6, Les tables. La grammaire du français par le menu, 251-264.

MULLER Claude, 2002, Les bases de la syntaxe. Syntaxe contrastive français-langues voisines, Pessac, Presses Universitaires de Bordeaux.

Ozouf Corinne, 2004, «Caractère différentiel et relation d'équivalence entre voir et regarder », Cahier du CRISCO 16, 1-25.

REY Alain \& CHANTREAU Sophie, 2003, « Préface », Dictionnaire d'expressions et locutions, Paris : Le Robert.

RIEGEl Martin, Pellat Jean-Christophe \& RIoul René, 2014 [1994], Grammaire méthodique du français, Paris : Presses Universitaires de France.

SENELLART Jean, 1998, « Reconnaissance automatique des entrées du lexique-grammaire des phrases figées ", Travaux de Linguistique 37, Le lexique-grammaire, 109-126.

SENELLART Jean, 1999, Localisation d'expressions linguistiques complexes dans de gros corpus, Thèse de doctorat, Université Paris 7.

SHINMURA Izuru (ed.), 2018 [1955], Kojiên, Japon : Iwamura.

SVENSSON Maria-Helena, 2004, Critères de figement. L'identification des expressions figées en français contemporain, Umeå : Umeå University. 
TANAKA Satoko, 1996, “The Polysemic Structure of 'miru (see)"', Gengo Kenkyu 110, Journal of the Linguistic Society of Japan, 120-142, also available at: https://www.jstage.jst.go.jp/article/ gengo1939/1996/110/1996_110_120/_article/-char/en

TANAKA Satoko, 1999, Shikaku doushi no imiron (Sémantique des verbes visuels), Thèse de doctorat en linguistique, Université de Nagoya, Nagoya, Japon.

VIGUIER Marie-Hélène \& GREZKA Aude (eds.), 2017, Figement en mouvement et complexité en devenir : regards sur quelques problèmes liés aux structures défigées et complexes, Revue ELA, Études de linguistique appliquée 186.

WILLEMS Dominique, 1983, « Regarde voir. Les verbes de perception visuelle et la complémentation verbale », in ROEGIEST Eugeen \& TASMOWSKI Liliane (eds.), Verbes et Phrases dans les Langues Romanes. Mélanges offerts à Louis Mourin, Gent : Romanica Gandensia, 147-158.

\section{NOTES}

1. Nous avons bénéficié du soutien financier de la Subvention pour les Recherches Scientifiques des jeunes chercheurs (B) du Ministère de l'Éducation et des Sciences du Japon (JSPS KAKENHI Grant Numbers 16K16822, représenté par A. Kijima) et du soutien du programme 2018 « Exploration Japon » (représenté par A. Grezka).

2. Les exemples en japonais sont présentés comme suit : la première ligne correspond à l'énoncé écrit en japonais. La deuxième ligne donne la prononciation de cet énoncé, annotée avec la méthode Hepburn (la transcription des mots japonais en alphabet latin) et la traduction française, entre parenthèses. Sur la troisième ligne, nous transcrivons l'énoncé japonais mot à mot en utilisant les termes équivalents en français. Les éléments soulignés sont les particules japonaises. Elles expriment la fonction des mots qui les précèdent. Les abréviations pour les particules sont les suivantes : $S$ : particule du sujet, sujet syntaxique ga; Th: particule thématique ha; 0 : particule de l'objet $o$; Loc: particule locative $n i ; D$ : particule de détermination no.

3. Notamment les travaux de D. Willems [1983]; J-J. Franckel [1989]; J-J. Franckel \& D. Lebaud [1990] ; J. Dubois \& F. Dubois-Charlier [1997] ; C. Ozouf [2004] ; etc.

4. Une liste rassemblant près de 1500 mots, les plus fréquents de la langue française, a été constituée par le lexicologue É. Brunet. Elle rend compte de la langue que lisent les élèves francophones. Ces mots, qui sont extraits de textes littéraires ou non, ont été ramenés à leur base lexicale. Elle est consultable et téléchargeable à partir du lien suivant : http:// eduscol.education.fr/cid50486/liste-de-frequence-lexical.html

5. Voir les travaux de H. Bat-Zeev Shyldkrot [1981 : 387-407 ; 1997 : 205-224], M. Riegel, J-C. Pellat \& R. Riou [2014 : 442-443], P. Le Goffic [1993 :166]. C. Muller [2002 : 229] parle de diathèse par auxiliation : «Le président du jury a remis à Marie le prix du premier roman / Marie s'est vu remettre le prix du premier roman (par le président du jury)».

6. Nous avons 307.238 occurrences de 見, 48.988 de 観, 36.360 de 視, 18.442 de 診, 8.807 de 看 et 8.838 de 覧 dans notre corpus National Institute for Japanese Language and Linguistics (NINJAL), du japonais moderne écrit.

7. Le dictionnaire japonais Kôjien [広辞苑, 2018] explique que mieru est construit sur la base du verbe miru en y ajoutant le verbe auxiliaire « yu » qui représente la volonté ou la spontanéité. 
8. Ces deux exemples sont cités par T. Miyajima [1972], la traduction française est la nôtre.

9. L'abréviation «CR » est utilisée pour « compte rendu » dans A. Grezka [2009].

10. Le TLFi (http://atilf.atilf.fr) est la version informatisée du TLF, un dictionnaire des XIXe et XXe siècles en 16 volumes et 1 supplément.

11. Le 仏和辞典 (futsu-wa Jiten) est un dictionnaire français-japonais collaboratif qui s'appuie sur la participation de ses membres pour enrichir en permanence sa base de données (http://9.dee.cc/ hakase2/).

12. À ne pas confondre avec avoir des yeux d'aigle. L'expression avoir un regard d'aigle désigne le fait d'avoir un esprit perspicace, clairvoyant et n'a donc rien à voir avec la perception visuelle.

\section{RÉSUMÉS}

Le figement représente un aspect fondamental du fonctionnement des langues et reste le meilleur témoin de leur vitalité. Il constitue un phénomène essentiel dans le fonctionnement du langage au point qu'on peut se demander s'il existe des assemblages réellement libres. Le nombre d'unités polylexicales dépasse largement celui des unités monolexicales appartenant à la même partie du discours. Le phénomène du figement ne se restreint pas au lexique puisqu'il a pratiquement le monopole de la grammaticalisation: il touche à la fois les domaines de la syntaxe, de la sémantique, du lexique et de la pragmatique. Dans cet article, nous allons nous intéresser au figement dans le cadre des verbes de perception en français et en japonais. Ces verbes sont très courants et importants dans le langage, en raison des phénomènes perceptifs qu'ils expriment. L'objet linguistique est ici restreint à l'étude des verbes de perception visuelle ( voir, regarder, etc.; miru, mieru, etc.). D'un point de vue extralinguistique, la vue est notre première source d'information - considérée comme objective - sur la réalité perçue. La majorité des informations que nous recevons passent par nos yeux. On comprend donc l'intérêt que présente la perception visuelle du point de vue linguistique. Le champ lexical relatif à la vue est significativement plus abondant que celui des autres sens.

Frozenness is a fundamental aspect of how languages function and remains the best indicator of their vitality. It is such an essential phenomenon in language functioning that one can wonder if free strings do exist. Polylexical units largely outnumber monolexical units belonging to the same parts of speech. The phenomenon of fixedness cannot be restricted to the lexicon since it has practically the monopoly of grammaticalization: it also concerns the domains of syntax, semantics and pragmatics. In this article, we are interested in fixedness within the context of perception verbs both in French and in Japanese. Such verbs are very frequent and important in language because of the perceptual phenomena they express. The linguistic object is here restricted to the study of visual perception verbs (voir, regarder, etc.; miru, mieru, etc.). From an extralinguistic point of view, sight is man's primary source of information - considered as objective - on perceived reality. The eyes are the main sensory organs providing information on the real, which explains why visual perception is highly interesting from a linguistic viewpoint. The lexical field relating to sight is significantly richer than that of the other senses. 
INDEX

Mots-clés : étude comparative, figement, français, japonais, perception, séquence figée, verbe, voir, regarder, phraséologie contrastive française-japonaise

Keywords : comparative study, frozenness, French, Japanese, perception, fixed phrase, verb, see, look / watch, French-Japanese contrastive phraseology

\section{AUTEURS}

\section{AUDE GREZKA}

CNRS \& Université de Paris 13, France

grezka@lipn.univ-paris13.fr

\section{AÏ KIJIMA}

Université de technologie de Chiba, Japon

ai.kijima@p.chibakoudai.jp 